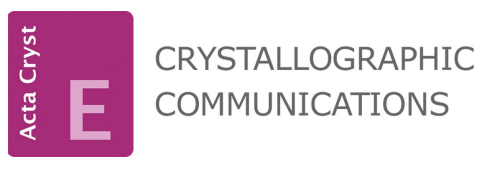

ISSN 2056-9890

Received 13 August 2018

Accepted 15 August 2018

Edited by W. T. A. Harrison, University of Aberdeen, Scotland

Keywords: crystal structure; D-mannose bisulfite adduct; sodium hydrogen sulfite; sodium metabisulfite.

CCDC reference: 1862155

Supporting information: this article has supporting information at journals.iucr.org/e

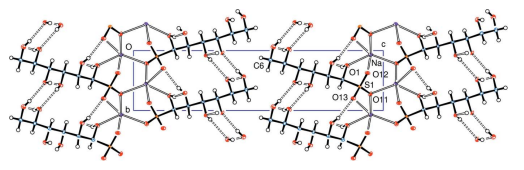

OPEN $\odot$ ACCESS

\section{Crystal structure of sodium (1S)-D-mannit-1-yl- sulfonate}

\author{
Alan H. Haines* and David L. Hughes* \\ School of Chemistry, University of East Anglia, Norwich, NR4 7TJ, UK. *Correspondence e-mail: a.haines@uea.ac.uk, \\ d.I.hughes@uea.ac.uk
}

The title salt, $\mathrm{Na}^{+} \cdot \mathrm{C}_{6} \mathrm{H}_{13} \mathrm{O}_{9} \mathrm{~S}^{-}$[systematic name: sodium $(1 S, 2 S, 3 S, 4 R, 5 R)$ 1,2,3,4,5,6-hexahydroxyhexane-1-sulfonate], is formed by reaction of D-mannose with sodium bisulfite (sodium hydrogen sulfite) in water. The anion has an openchain structure with the $\mathrm{S}$ atom and the $\mathrm{C}$ atoms of the carbohydrate chain forming an essentially planar zigzag chain in which the absolute values of the torsion angles lie between $173.6(2)$ and $179.9(3)^{\circ}$. The sodium cations are penta-coordinated by $\mathrm{O}$ atoms, with one link to a carbohydrate $\mathrm{O}$ atom and four to $\mathrm{O}$ atoms of sulfonate residues in separate anions, thus creating a threedimensional network. The carbohydrate anions are arranged in a head $\left(-\mathrm{SO}_{3}{ }^{-}\right)$ to head $\left(-\mathrm{SO}_{3}{ }^{-}\right)$arrangement, thereby forming two parallel sheets linked through coordination to sodium ions, with each sheet containing intermolecular hydrogen bonds between the anionic residues. Unusually, the double sheets are not connected to neighbouring sets of double sheets, either by ion coordination or intermolecular hydrogen bonding.

\section{Chemical context}

Adducts formed by the reaction of aldehydes and bisulfite anions have long been used for aldehyde purification since they are often crystalline, whereas the parent aldehydes are often liquids with varying stabilities on storage. The addition reaction is reversible, which makes the bisulfite compounds useful intermediates in the synthesis of other adducts from aldehydes, such as cyanohydrins. Further, bisulfite adducts are useful since they are soluble in water, which can be important if the compounds need to be compatible with aqueous, biological systems, for example to aid delivery of medicinal drugs insoluble in water. Such considerations are important since aldehydes are involved in many synthetic processes for the production of commercially relevant compounds, including pharmaceuticals, and the relative advantages of using different counter-ions (e.g. stability, hygroscopicity, ease of filtration of the adduct) are of interest (Kissane et al., 2013).

The bisulfite addition products of aldoses are unusual in that they are acyclic compounds despite the fact that the parent carbohydrates exist predominantly in the cyclic, hemiacetal form. Although such adducts were synthesised many years ago, unequivocal proof of their acyclic nature awaited $\mathrm{X}$-ray structure determination, firstly on the potassium adducts of D-glucose and D-mannose (Cole et al., 2001), of D-galactose (Haines \& Hughes, 2010), D-ribose (Haines \& Hughes, 2014), D-lyxose (Haines \& Hughes, 2015), and of the sodium adducts of D-glucose (Haines \& Hughes, 2012) and D-lyxose (Haines \& Hughes, 2016). 
We now report the preparation, properties, and crystal structure of the sodium bisulfite adduct of D-mannose, and comment on its significant structural difference from that of the corresponding potassium adduct.

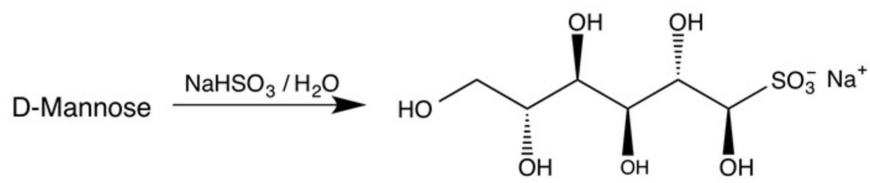

Mixing concentrated, equimolar solutions of D-mannose and sodium bisulfite (sodium hydrogen sulfite formed by the in situ hydrolysis of sodium metabisulfite) in water led to immediate precipitation of the adduct in high yield; this was purified by recrystallization from water, giving material stable in air but which melted over a large temperature range (413$444 \mathrm{~K}$ ) with extended and continual decomposition.

Obtaining suitable crystals for X-ray analysis was challenging since there was a tendency for formation of thin, rough, multiple crystals, but slow crystallization at approximately $283-286 \mathrm{~K}$ and careful selection from the crop soproduced afforded the crystal for examination. The newly formed chiral centre at $\mathrm{C} 1$ had the $S$-configuration and in solution in water:acetic acid (9:1) the adduct gave a positive rotation which remained stable over an extended period, suggesting that hydrolysis to its component parts was hindered under the acidic conditions.

\section{Structural commentary}

The newly formed chiral centre at $\mathrm{C} 1$ has the $S$-configuration (as shown in Fig. 1) and the systematic name for the salt is sodium $\quad(1 S, 2 S, 3 S, 4 R, 5 R)-1,2,3,4,5,6$-hexahydroxyhexane-1sulfonate. The anion has an open-chain structure in which the $\mathrm{S}$ atom and the $\mathrm{C}$ atoms of the sugar chain form an essentially planar zigzag (all-trans) chain with the corresponding torsion angles lying between the absolute values of 173.6 (2) and $179.9(3)^{\circ}$. The hydrogen atoms of the hydroxyl groups on $\mathrm{C} 1$ to $\mathrm{C} 6$ of the carbon chain form hydrogen bonds with oxygen atoms $\mathrm{O} 2$, O13, O5, O3, O6 and O4, respectively, of neighbouring chains (Fig. 1 and Table 1) and all the hydroxyl $\mathrm{O}$ atoms except $\mathrm{O} 1$ are acceptors of hydrogen bonds; $\mathrm{O} 1$ is bonded to a sodium ion. We note that all the hydrogen bonds are arranged in cyclic systems, some comprising four $\mathrm{O}-$ $\mathrm{H} \cdots \mathrm{O}$ bonds, others with two $\mathrm{O}-\mathrm{H} \cdots \mathrm{O}$ bonds plus two $\mathrm{Na}-$ O coordination bonds.

Unusually, the sodium atom has a coordination sphere of five rather than six oxygen atoms, hexa-coordination having been observed in related adducts from D-glucose (Haines \& Hughes, 2012) and D-lyxose (Haines \& Hughes, 2016). Further, coordination of a sodium ion by $\mathrm{O} 1$ of the carbohydrate chain and oxygen atoms $\mathrm{O} 11, \mathrm{O} 12$ and $\mathrm{O} 13$ of four different sulfonate groups leads to a sheet of $\mathrm{Na}$ ions coordinated to the 'heads' of the anions, Fig. 2.
Table 1

Hydrogen-bond geometry $\left(\AA,{ }^{\circ}\right)$.

\begin{tabular}{|c|c|c|c|c|}
\hline$D-\mathrm{H} \cdots A$ & $D-\mathrm{H}$ & $\mathrm{H} \cdots A$ & $D \cdots A$ & $D-\mathrm{H} \cdots A$ \\
\hline $\mathrm{O} 1-\mathrm{H} 1 O \cdots \mathrm{O} 2^{\mathrm{ii}}$ & $0.81(3)$ & $1.92(4)$ & $2.693(4)$ & $160(8)$ \\
\hline $\mathrm{O} 2-\mathrm{H} 2 \mathrm{O} \cdots \mathrm{O} 1$ & $0.81(3)$ & $2.36(5)$ & $2.828(3)$ & $118(5)$ \\
\hline $\mathrm{O} 2-\mathrm{H} 2 O \cdots \mathrm{O} 13^{\mathrm{i}}$ & $0.81(3)$ & $2.08(4)$ & $2.745(4)$ & $140(5)$ \\
\hline $\mathrm{O} 3-\mathrm{H} 3 O \cdots \mathrm{O} 4$ & $0.81(3)$ & $2.32(7)$ & $2.838(3)$ & $123(7)$ \\
\hline $\mathrm{O} 3-\mathrm{H} 3 O \cdots \mathrm{O}^{\mathrm{v}}$ & $0.81(3)$ & $2.11(6)$ & $2.743(4)$ & $136(7)$ \\
\hline $\mathrm{O} 4-\mathrm{H} 4 O \cdots \mathrm{O}^{\mathrm{vi}}$ & $0.82(3)$ & $1.87(3)$ & $2.692(3)$ & $179(8)$ \\
\hline $\mathrm{O} 5-\mathrm{H} 5 \mathrm{O} \cdots \mathrm{O} 6^{\mathrm{ii}}$ & $0.80(3)$ & $1.91(3)$ & $2.704(4)$ & $169(6)$ \\
\hline $\mathrm{O} 6-\mathrm{H} 6 \mathrm{O} \cdots \mathrm{O} 4^{\mathrm{i}}$ & $0.81(3)$ & $2.00(4)$ & $2.753(4)$ & $154(6)$ \\
\hline $\mathrm{O} 6-\mathrm{H} 6 \mathrm{O} \cdots \mathrm{O} 5$ & $0.81(3)$ & $2.45(6)$ & $2.853(4)$ & $112(5)$ \\
\hline
\end{tabular}

Symmetry codes: (i) $x, y-1, z$; (ii) $x-1, y, z$; (v) $x, y+1, z$; (vi) $x+1, y, z$.

Table 2

Selected bond lengths $(\AA)$.

\begin{tabular}{llll}
\hline $\mathrm{Na}-\mathrm{O} 11^{\mathrm{i}}$ & $2.293(3)$ & $\mathrm{Na}-\mathrm{O} 1$ & $2.386(3)$ \\
$\mathrm{Na}-\mathrm{O} 12^{\text {ii }}$ & $2.328(3)$ & $\mathrm{Na}-\mathrm{O} 13^{\text {iv }}$ & $2.421(3)$ \\
$\mathrm{Na}-\mathrm{O} 11^{\text {iii }}$ & $2.370(3)$ & $\mathrm{Na}-\mathrm{O} 12^{\text {iii }}$ & $2.757(3)$ \\
\hline
\end{tabular}

Symmetry codes: (i) $x, y-1, z$; (ii) $x-1, y, z$; (iii) $-x+1, y-\frac{1}{2},-z+2$; (iv) $x-1, y-1, z$

The $\mathrm{Na}-\mathrm{O}$ bonds have lengths in the range 2.293 (3) to 2.421 (3) $\AA$ (Table 2) and form a distorted square pyramidal shape with $\mathrm{O} 1$ in the apical site. The next shortest $\mathrm{Na}-\mathrm{O}$ contact distance is $2.757 \AA$ to $\mathrm{O} 12^{\mathrm{iii}}$, which would provide a rather distorted octahedral coordination. Twofold screw axes of symmetry (parallel to the $b$ axis) relate the sodium ions in the sheet close to the $a b$ plane at $z=0$ and 1 , and the zigzag $C_{6}$ chains lie approximately normal to this plane and nearly parallel to the $c$ axis. The 'tails' of these chains, around the C6,O6 groups, lie close to the $z=\frac{1}{2}$ plane where the screw axes relate them to the tails of adjacent molecules. But, whereas the heads are linked through the sodium atoms across the $z=0$

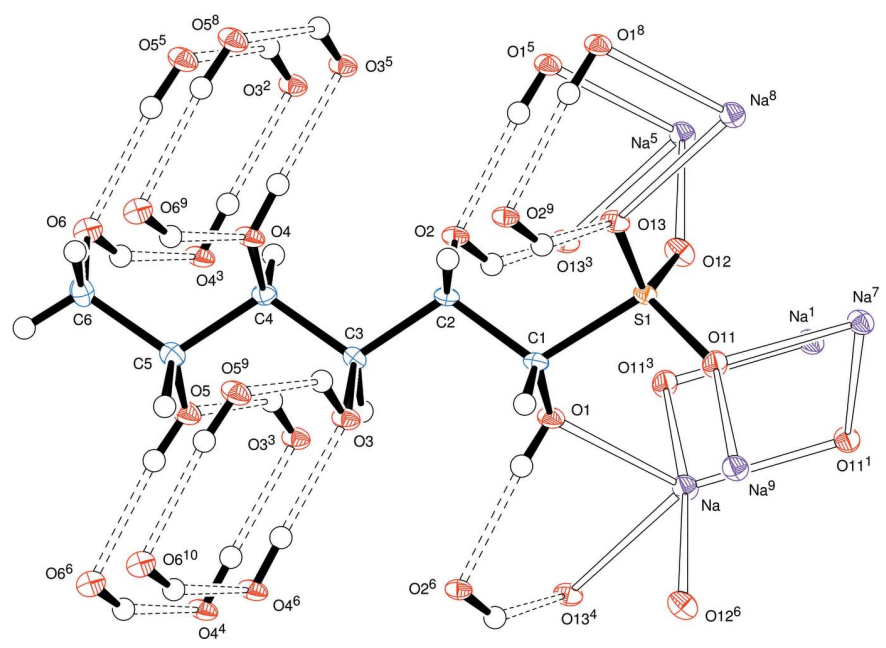

Figure 1

View of the D-mannose- $\mathrm{NaHSO}_{3}$ adduct, indicating the atom-numbering scheme. All sodium coordination contacts and hydrogen bonds involving the atoms of the sugar adduct are indicated. Displacement ellipsoids are drawn at the $50 \%$ probability level. Symmetry codes: (1) $1-x, y-\frac{1}{2}$, $2-z$; (2) $1+x, y-1, z$; (3) $x, y-1, z$; (4) $x-1, y-1, z ;$ (5) $1+x, y, z$; (6) $x-1, y, z ;$ (7) $1-x, \frac{1}{2}+y, 2-z$;(8) $1+x, 1+y, z$; (9) $x, 1+y, z ;$ (10) $x-1$, $1+y, z$. 


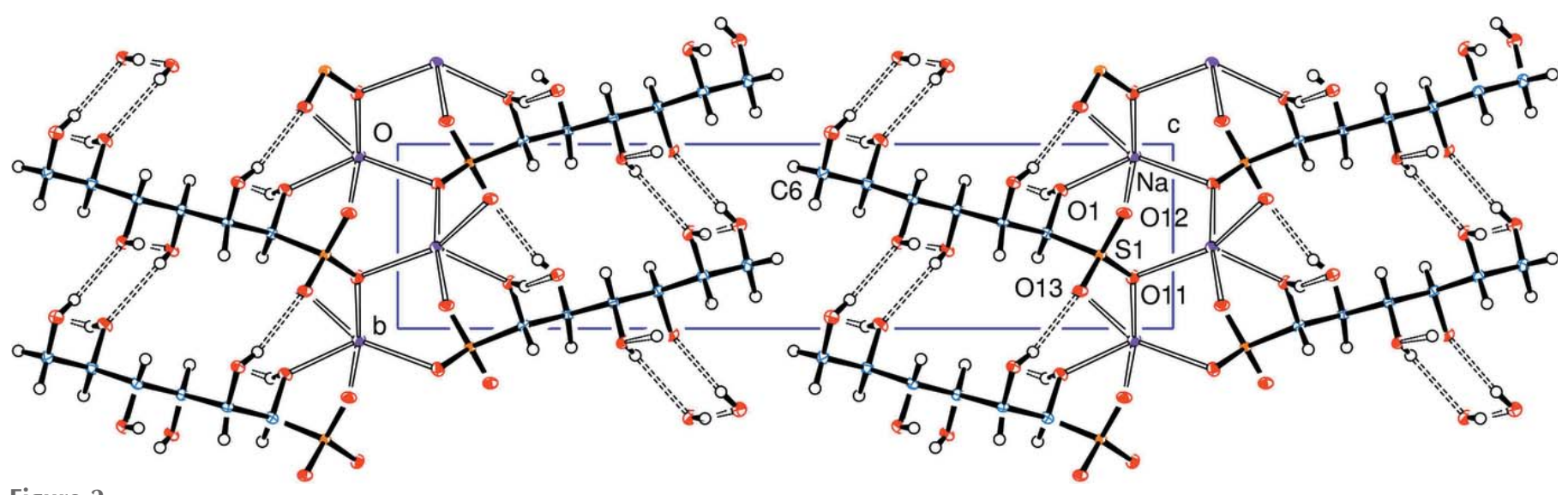

Figure 2

View down the $a$ axis of the crystal packing.

plane, there are no short intermolecular contacts across the $z=\frac{1}{2}$ plane; the shortest contacts here are $\mathrm{H} 61 \cdots \mathrm{H} 62^{11}=$

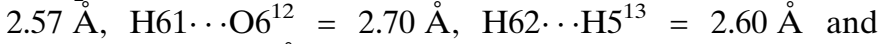
H62 $\cdots \mathrm{C}^{13}=2.95 \AA$, i.e. at normal van der Waals' distances; symmetry codes: (11) $1-x, y+\frac{1}{2}, 1-z$; (12) $2-x, y+\frac{1}{2}, 1-z$; (13) $1-x, y-\frac{1}{2}, 1-z$.

The neighbours of the zigzag $C_{6}$ chains are related only by translation parallel to the $a$ and $b$ axes, Fig. 3; all the cations here are aligned in the same direction. Anions related across the Na coordination plane and about the $z=\frac{1}{2}$ plane have the opposite alignment. Here, we observe a major difference between this sodium complex and the corresponding potassium D-mannose complex (Cole et al., 2001) where each $C_{6}$ chain is surrounded by four chains pointing in the opposite direction, as shown in Fig. 4. Hence the distances between the

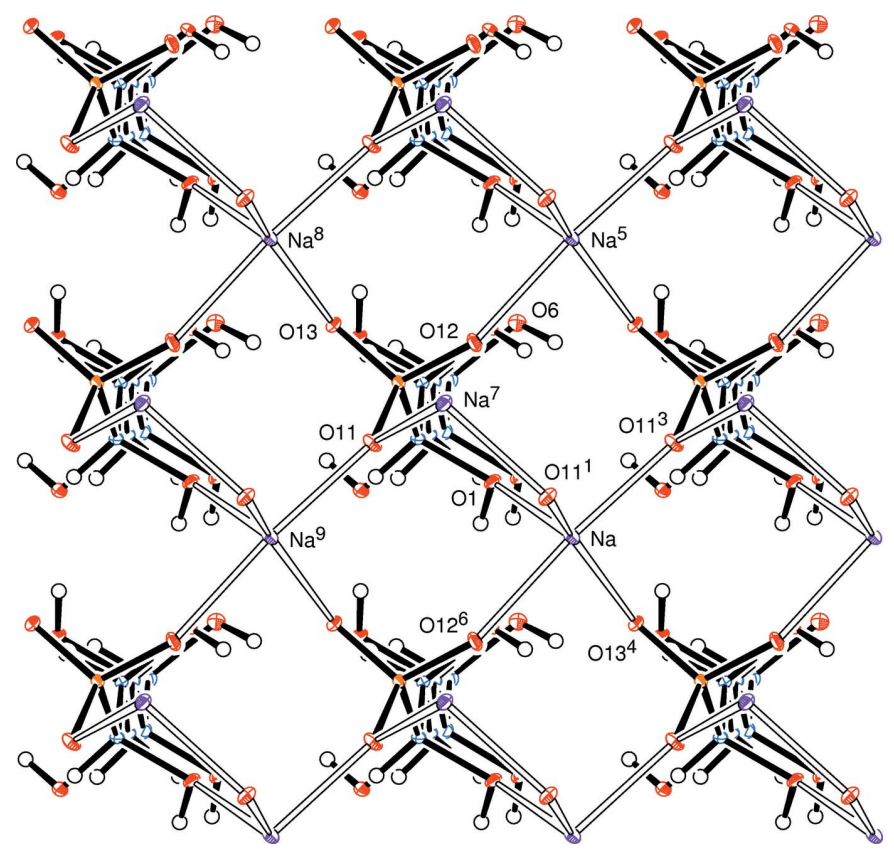

Figure 3

View looking along the all-trans sugar chain and neighbouring chains, all pointing in the same direction. Displacement ellipsoids are shown at the $30 \%$ probability level. Symmetry codes are defined as for Fig. 1 . coordination planes are quite different, viz $21.08 \AA$ in the sodium complex, but $11.55 \AA$ for the potassium compound.

\section{Supramolecular features}

A three-dimensional bonding network exists in the crystal structure through (i) penta-coordination of a sodium cation with oxygens from five different mannose bisulfite residues, and (ii) intermolecular hydrogen bonds from each of the six hydroxyl groups to acceptor oxygen atoms in four different residues.

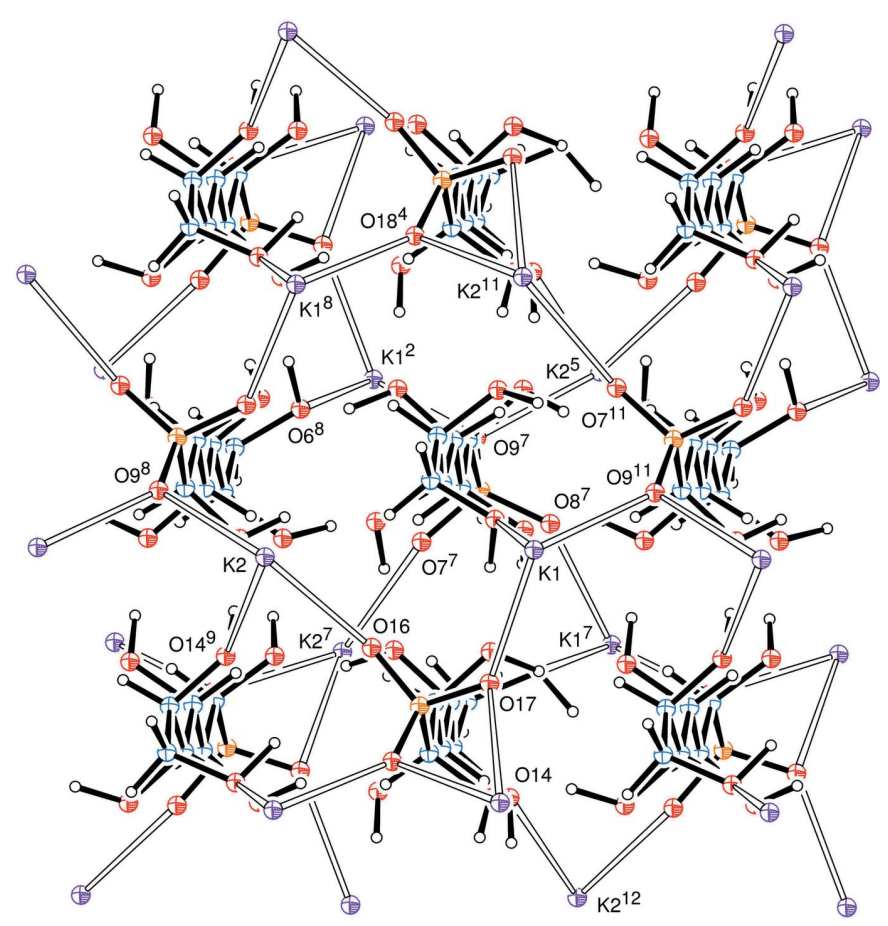

Figure 4

View looking along the all-trans sugar chain and neighbouring chains in the D-mannose-potassium bisulfite adduct; adjacent chains point in opposing directions. Atom coordinates were taken from the CCDC deposition, code 172060 (Cole et al., 2001). Atoms are represented by small spheres of arbitrary radii. Symmetry codes: (2) $1-x, \frac{1}{2}+y,-z ;$ (4) $1+x, y, z ;(5) 1-x, y-\frac{1}{2},-z$; (7) $x, y, z-1$; (8) $1-x, \frac{1}{2}+y, 1-z ;(9)-x$, $\frac{1}{2}+y,-z ;(11) 1-x, y-\frac{1}{2}, 1-z ;(12)-x, y-\frac{1}{2},-z$. 


\section{Spectroscopic results}

High resolution mass spectrometry in negative ion mode showed, as the base peak in the spectrum, a peak for $\left(\left[\mathrm{C}_{6} \mathrm{H}_{13} \mathrm{O}_{9} \mathrm{~S}_{1}\right]^{-}\right)$at $m / z 261.0287$ and a significant peak was observed at $m / z 243.0182\left(\left[\mathrm{C}_{6} \mathrm{H}_{13} \mathrm{O}_{9} \mathrm{~S}_{1}-\mathrm{H}_{2} \mathrm{O}\right]^{-}\right)$. A minor peak observed at $m / z \quad 359.1194\left(\left[\mathrm{C}_{12} \mathrm{H}_{23} \mathrm{O}_{12}\right]^{-}\right)$was assigned to a dimer ion $\left([2 M-\mathrm{H}]^{-}\right)$produced by association of a D-mannose molecule $\left(M=\mathrm{C}_{6} \mathrm{H}_{12} \mathrm{O}_{6}\right)$ with the mono-anion of D-mannose $\left(\left[\mathrm{C}_{6} \mathrm{H}_{11} \mathrm{O}_{6}\right]^{-}\right)$under the electrospray ionization conditions of the mass spectrometric measurement.

The ${ }^{1} \mathrm{H}$ NMR spectrum of the adduct in $\mathrm{D}_{2} \mathrm{O}$ indicated the presence of the $\alpha$ - and $\beta$-pyranose forms of D-mannose and the major and minor forms of the acyclic sulfonate in the \% ratios 25.24:13.14:55.00:6.62, respectively. Clearly, the $R$ stereoisomer at $\mathrm{C} 1$ is present in solution but only the $S$-isomer crystallizes. Further, some hydrolysis of the adduct to afford the parent sugar occurs during the NMR measurement.

The ${ }^{13} \mathrm{C}$ NMR spectrum showed signals for $\mathrm{C} 1$ nuclei at $\delta_{\mathrm{C}}$ 94.70, 94.31, 84.43 and 82.34 arising, respectively, from the $\alpha$ and $\beta$-pyranose forms of D-mannose, the minor adduct and the major adduct, in the \% ratios of 32.73:15.00:3.64:48.63.

\section{Synthesis and crystallization}

D-Mannose $(0.9 \mathrm{~g})$ was dissolved in water $(2 \mathrm{ml})$, sodium metabisulfite $(0.475 \mathrm{~g})$ was added, and the solution was then warmed to achieve complete solution. On cooling to room temperature, precipitation occurred within $3 \mathrm{~min}$ (see scheme). The product was collected by filtration, and dried to give the adduct (1.42 $\mathrm{g}, 84 \%$ ), a portion of which was recrystallized to afford the analytical sample, m.p. 413-444 K (with extended and continual decomposition); $[\alpha]_{\mathrm{D}}{ }^{20}+8.2$ (15 min.) (c, 0.79 in 9:1 $\mathrm{H}_{2} \mathrm{O}$ : HOAc). ${ }^{1} \mathrm{H}$ NMR $\left(\mathrm{D}_{2} \mathrm{O}, 400 \mathrm{MHz}\right.$, reference $M e_{3} \mathrm{COH}$ at $\left.\delta_{\mathrm{H}} 1.24\right): \delta_{\mathrm{H}} 5.17\left(d, J_{1,2}=1.5 \mathrm{~Hz}, \mathrm{H}-1\right.$ of $\alpha$ pyranose), 4.89 ( $d, J_{1,2}=0.8 \mathrm{~Hz}, \mathrm{H}-1$ of $\beta$-pyranose); signals for the major acyclic sulfonate: $\delta_{\mathrm{H}} 4.64(s, \mathrm{H}-1), 4.20\left(d, J_{2,3}=\right.$ $9.5 \mathrm{~Hz}, \mathrm{H}-2)$; for the minor acyclic sulfonate: $\delta_{\mathrm{H}} 4.64\left(d, J_{1,2}=\right.$ $5.2 \mathrm{~Hz}, \mathrm{H}-1), 4.07\left(d, J_{2,3}=7.8 \mathrm{~Hz}, \mathrm{H}-2\right)$; ratio of major to minor sulfonate $=8.3: 1 .{ }^{13} \mathrm{C}$ NMR $\left(\mathrm{D}_{2} \mathrm{O}, 100 \mathrm{MHz}\right.$, reference $M e_{3} \mathrm{COH}$ at $\left.\delta_{\mathrm{C}} 30.29\right): \delta_{\mathrm{C}} 94.70$ (C1, $\alpha$-pyranose), 94.31 (C1, $\beta$ pyranose); signals for the major acyclic sulfonate: $\delta_{\mathrm{C}} 82.34$ (C1), 71.48, 69.46, 69.18*, 68.91* (C2, C3, C4, C5), 63.96 (C6); the minor acyclic sulfonate showed a peak at $\delta_{\mathrm{C}} 84.43$ (C1). Each of the signals marked with * is the average value of two closely spaced singlets of equal intensity separated by $4 \mathrm{~Hz}$. The reasons for these small separations in the proton decoupled ${ }^{13} \mathrm{C}$ spectrum are not clear.

Integration of the various signals for $\mathrm{H}-1$ in the ${ }^{1} \mathrm{H}$ NMR spectrum indicated that the species $\alpha$-pyranose, $\beta$-pyranose, major acyclic sulfonate and minor acyclic sulfonate were present in the \% ratios of 25.24: $13.14: 55.00: 6.62$, respectively. In the ${ }^{13} \mathrm{C}$ NMR spectrum, based on peak heights, the corresponding ratios were: 32.73: 15.00: 48.63: 3.64.

HRESMS (negative ion mode, measured in an $\mathrm{H}_{2} \mathrm{O} / \mathrm{MeOH}$, solution) gave a base peak at $m / z 261.0287\left(\left[\mathrm{C}_{6} \mathrm{H}_{13} \mathrm{O}_{9} \mathrm{~S}_{1}\right]^{-}\right)$, and a significant peak at $243.0182\left(\left[\mathrm{C}_{6} \mathrm{H}_{13} \mathrm{O}_{9} \mathrm{~S}_{1}-\mathrm{H}_{2} \mathrm{O}\right]^{-}\right)$.
Table 3

Experimental details.

\begin{tabular}{|c|c|}
\hline \multicolumn{2}{|l|}{ Crystal data } \\
\hline Chemical formula & $\mathrm{Na}^{+} \cdot \mathrm{C}_{6} \mathrm{H}_{13} \mathrm{NaO}_{9} \mathrm{~S}^{-}$ \\
\hline$M_{\mathrm{r}}$ & 284.21 \\
\hline Crystal system, space group & Monoclinic, $P 2_{1}$ \\
\hline Temperature (K) & 140 \\
\hline$a, b, c(\AA)$ & $4.8744(2), 5.0042(2), 21.0759(10)$ \\
\hline$\beta\left({ }^{\circ}\right)$ & $93.867(4)$ \\
\hline$V\left(\mathrm{~A}^{3}\right)$ & $512.92(4)$ \\
\hline$Z$ & 2 \\
\hline Radiation type & Мo $K \alpha$ \\
\hline$\mu\left(\mathrm{mm}^{-1}\right)$ & 0.40 \\
\hline Crystal size $(\mathrm{mm})$ & $0.52 \times 0.20 \times 0.04$ \\
\hline \multicolumn{2}{|l|}{ Data collection } \\
\hline Diffractometer & $\begin{array}{l}\text { Oxford Diffraction Xcalibur 3/ } \\
\text { Sapphire3 CCD }\end{array}$ \\
\hline Absorption correction & $\begin{array}{l}\text { Multi-scan (CrysAlis PRO; } \\
\text { Agilent, 2014) }\end{array}$ \\
\hline$T_{\min }, T_{\max }$ & $0.618,1.000$ \\
\hline $\begin{array}{l}\text { No. of measured, independent and } \\
\text { observed }[I>2 \sigma(I)] \text { reflections }\end{array}$ & $9424,2972,2926$ \\
\hline$R_{\text {int }}$ & 0.030 \\
\hline$(\sin \theta / \lambda)_{\max }\left(\AA^{-1}\right)$ & 0.703 \\
\hline \multicolumn{2}{|l|}{ Refinement } \\
\hline$R\left[F^{2}>2 \sigma\left(F^{2}\right)\right], w R\left(F^{2}\right), S$ & $0.039,0.093,1.16$ \\
\hline No. of reflections & 2972 \\
\hline No. of parameters & 206 \\
\hline No. of restraints & 7 \\
\hline $\mathrm{H}$-atom treatment & All $\mathrm{H}$-atom parameters refined \\
\hline$\Delta \rho_{\max }, \Delta \rho_{\min }\left(\mathrm{e} \AA^{-3}\right)$ & $0.49,-0.45$ \\
\hline Absolute structure & $\begin{array}{l}\text { Flack } x \text { determined using } 1240 \\
\quad \text { quotients }\left[\left(I^{+}\right)-\left(I^{-}\right)\right] /\left[\left(I^{+}\right)+\left(I^{-}\right)\right] \\
\text {(Parsons } \text { et al., } 2013)\end{array}$ \\
\hline Absolute structure parameter & $0.04(4)$ \\
\hline
\end{tabular}

Computer programs: CrysAlis PRO (Agilent, 2014), SHELXT (Sheldrick, 2015a), SHELXL2014/7 (Sheldrick, 2015b), ORTEP (Johnson, 1976) and ORTEP-3 for Windows and WinGX (Farrugia, 2012).

\section{Refinement}

Crystal data, data collection and structure refinement details are summarized in Table 3. All the hydrogen atoms were located in difference maps and were refined with isotropic thermal parameters; the hydroxyl hydrogen atoms were refined with constrained $\mathrm{O}-\mathrm{H}$ distances.

\section{Acknowledgements}

We thank the EPSRC UK National Mass Spectrometry Facility (NMSF) at Swansea University for determination of the low and high resolution mass spectra, Dr Sergey Nepogodiev of the John Innes Centre, Norwich and Dr Ryan Tinson of the University of East Anglia for measurement of the NMR spectra, and Dr James Orton of the UK National Crystallographic Service for preliminary measurements.

\section{References}

Agilent (2014). CrysAlis PRO. Agilent Technologies Ltd, Yarnton, England.

Cole, E. R., Craig, D. C., Fitzpatrick, L. J., Hibbert, D. B. \& Stevens, J. D. (2001). Carbohydr. Res. 335, 1-10.

Farrugia, L. J. (2012). J. Appl. Cryst. 45, 849-854. 
Haines, A. H. \& Hughes, D. L. (2010). Carbohydr. Res. 345, 27052708.

Haines, A. H. \& Hughes, D. L. (2012). Acta Cryst. E68, m377-m378. Haines, A. H. \& Hughes, D. L. (2014). Acta Cryst. E70, 406-409. Haines, A. H. \& Hughes, D. L. (2015). Acta Cryst. E71, 993-996. Haines, A. H. \& Hughes, D. L. (2016). Acta Cryst. E72, 628-631. Johnson, C. K. (1976). ORTEPII. Report ORNL-5138. Oak Ridge National Laboratory, Tennessee, USA.
Kissane, M. G., Frank, S. A., Rener, G. A., Ley, C. P., Alt, C. A., Stroud, P. A., Vaid, R. K., Boini, S. K., McKee, L. A., Vicenzi, J. T. \& Stephenson, G. A. (2013). Tetrahedron Lett. 54, 6587-6591.

Parsons, S., Flack, H. D. \& Wagner, T. (2013). Acta Cryst. B69, 249 259.

Sheldrick, G. M. (2015a). Acta Cryst. A71, 3-8.

Sheldrick, G. M. (2015b). Acta Cryst. C71, 3-8. 


\section{supporting information}

Acta Cryst. (2018). E74, 1314-1318 [https://doi.org/10.1107/S2056989018011556]

\section{Crystal structure of sodium (1S)-D-mannit-1-ylsulfonate}

\section{Alan H. Haines and David L. Hughes}

Computing details

Data collection: CrysAlis PRO (Agilent, 2014); cell refinement: CrysAlis PRO (Agilent, 2014); data reduction: CrysAlis PRO (Agilent, 2014); program(s) used to solve structure: SHELXT (Sheldrick, 2015a); program(s) used to refine structure: SHELXL2014/7 (Sheldrick, 2015b); molecular graphics: ORTEP (Johnson, 1976) and ORTEP-3 for Windows (Farrugia, 2012); software used to prepare material for publication: SHELXL2014/7 (Sheldrick, 2015b) and WinGX

(Farrugia, 2012).

Sodium $(1 S, 2 S, 3 S, 4 R, 5 R)-1,2,3,4,5,6-\backslash$ hexahydroxyhexane-1-sulfonate

\section{Crystal data}

$\mathrm{Na}^{+} \cdot \mathrm{C}_{6} \mathrm{H}_{13} \mathrm{NaO}_{9} \mathrm{~S}^{-}$

$M_{r}=284.21$

Monoclinic, $P 2_{1}$

$a=4.8744(2) \AA$

$b=5.0042(2) \AA$

$c=21.0759(10) \AA$

$\beta=93.867(4)^{\circ}$

$V=512.92(4) \AA^{3}$

$Z=2$

\section{Data collection}

Oxford Diffraction Xcalibur 3/Sapphire3 CCD diffractometer

Radiation source: Enhance (Mo) X-ray Source

Graphite monochromator

Detector resolution: 16.0050 pixels $\mathrm{mm}^{-1}$

Thin slice $\varphi$ and $\omega$ scans

Absorption correction: multi-scan

(CrysAlis PRO; Agilent, 2014)

$T_{\min }=0.618, T_{\max }=1.000$

\section{Refinement}

Refinement on $F^{2}$

Least-squares matrix: full

$R\left[F^{2}>2 \sigma\left(F^{2}\right)\right]=0.039$

$w R\left(F^{2}\right)=0.093$

$S=1.16$

2972 reflections

206 parameters

7 restraints

Primary atom site location: dual

Hydrogen site location: difference Fourier map
$F(000)=296$

$D_{\mathrm{x}}=1.840 \mathrm{Mg} \mathrm{m}^{-3}$

Mo $K \alpha$ radiation, $\lambda=0.71073 \AA$

Cell parameters from 4715 reflections

$\theta=3.9-32.5^{\circ}$

$\mu=0.40 \mathrm{~mm}^{-1}$

$T=140 \mathrm{~K}$

Plate, colourless

$0.52 \times 0.20 \times 0.04 \mathrm{~mm}$

9424 measured reflections

2972 independent reflections

2926 reflections with $I>2 \sigma(I)$

$R_{\text {int }}=0.030$

$\theta_{\max }=30.0^{\circ}, \theta_{\min }=3.9^{\circ}$

$h=-6 \rightarrow 6$

$k=-7 \rightarrow 7$

$l=-29 \rightarrow 29$

All $\mathrm{H}$-atom parameters refined

$w=1 /\left[\sigma^{2}\left(F_{0}^{2}\right)+(0.0295 P)^{2}+0.694 P\right]$

where $P=\left(F_{\mathrm{o}}{ }^{2}+2 F_{\mathrm{c}}{ }^{2}\right) / 3$

$(\Delta / \sigma)_{\max }<0.001$

$\Delta \rho_{\max }=0.49 \mathrm{e} \AA^{-3}$

$\Delta \rho_{\min }=-0.44$ e $\AA^{-3}$

Absolute structure: Flack $x$ determined using

1240 quotients [(I+)-(I-)]/[(I+)+(I-)] (Parsons et al., 2013)

Absolute structure parameter: 0.04 (4) 


\section{Special details}

Geometry. All esds (except the esd in the dihedral angle between two 1.s. planes) are estimated using the full covariance matrix. The cell esds are taken into account individually in the estimation of esds in distances, angles and torsion angles; correlations between esds in cell parameters are only used when they are defined by crystal symmetry. An approximate (isotropic) treatment of cell esds is used for estimating esds involving l.s. planes.

Fractional atomic coordinates and isotropic or equivalent isotropic displacement parameters $\left(\hat{A}^{2}\right)$

\begin{tabular}{lllll}
\hline & $x$ & $y$ & $z$ & $U_{\text {iso }} * / U_{\mathrm{eq}}$ \\
\hline $\mathrm{C} 1$ & $0.5658(7)$ & $0.4844(7)$ & $0.83763(16)$ & $0.0099(6)$ \\
$\mathrm{O} 1$ & $0.4257(5)$ & $0.2507(5)$ & $0.85501(12)$ & $0.0124(5)$ \\
$\mathrm{C} 2$ & $0.7379(7)$ & $0.4250(7)$ & $0.78081(16)$ & $0.0092(6)$ \\
$\mathrm{O} 2$ & $0.9236(5)$ & $0.2083(5)$ & $0.79422(12)$ & $0.0112(5)$ \\
$\mathrm{C} 3$ & $0.5507(7)$ & $0.3570(7)$ & $0.72169(16)$ & $0.0103(6)$ \\
$\mathrm{O} 3$ & $0.3628(5)$ & $0.5738(6)$ & $0.70848(11)$ & $0.0124(5)$ \\
$\mathrm{C} 4$ & $0.7176(7)$ & $0.2973(7)$ & $0.66432(16)$ & $0.0106(6)$ \\
$\mathrm{O} 4$ & $0.8619(5)$ & $0.5330(5)$ & $0.64719(12)$ & $0.0132(5)$ \\
$\mathrm{C} 5$ & $0.5343(7)$ & $0.2160(7)$ & $0.60581(16)$ & $0.0121(6)$ \\
$\mathrm{O} 5$ & $0.3884(5)$ & $-0.0177(5)$ & $0.62226(13)$ & $0.0149(5)$ \\
$\mathrm{C} 6$ & $0.6989(8)$ & $0.1565(7)$ & $0.54832(17)$ & $0.0142(7)$ \\
$\mathrm{O} 6$ & $0.8875(5)$ & $-0.0609(5)$ & $0.55969(13)$ & $0.0150(5)$ \\
$\mathrm{S} 1$ & $0.77100(15)$ & $0.60171(15)$ & $0.90636(4)$ & $0.00914(16)$ \\
$\mathrm{O} 11$ & $0.5782(5)$ & $0.7211(6)$ & $0.94817(12)$ & $0.0139(5)$ \\
$\mathrm{O} 12$ & $0.9110(5)$ & $0.3741(5)$ & $0.93700(12)$ & $0.0142(5)$ \\
$\mathrm{O} 13$ & $0.9575(5)$ & $0.7966(5)$ & $0.88046(12)$ & $0.0125(5)$ \\
$\mathrm{Na}$ & $0.2652(3)$ & $0.0627(3)$ & $0.94976(6)$ & $0.0129(3)$ \\
$\mathrm{H} 1$ & $0.436(10)$ & $0.615(13)$ & $0.825(2)$ & $0.024(13)^{*}$ \\
$\mathrm{H} 2$ & $0.832(11)$ & $0.605(16)$ & $0.776(3)$ & $0.037(15)^{*}$ \\
$\mathrm{H} 3$ & $0.412(9)$ & $0.198(9)$ & $0.732(2)$ & $0.007(10)^{*}$ \\
$\mathrm{H} 4$ & $0.839(8)$ & $0.167(8)$ & $0.6749(19)$ & $0.002(9)^{*}$ \\
$\mathrm{H} 5$ & $0.377(10)$ & $0.363(11)$ & $0.595(2)$ & $0.020(13)^{*}$ \\
$\mathrm{H} 61$ & $0.800(9)$ & $0.326(9)$ & $0.5382(19)$ & $0.004(10)^{*}$ \\
$\mathrm{H} 62$ & $0.588(9)$ & $0.122(11)$ & $0.511(2)$ & $0.015(11)^{*}$ \\
$\mathrm{H} 1 \mathrm{O}$ & $0.281(8)$ & $0.275(15)$ & $0.834(3)$ & $0.045(18)^{*}$ \\
$\mathrm{H} 2 \mathrm{O}$ & $0.857(10)$ & $0.118(11)$ & $0.821(2)$ & $0.025(13)^{*}$ \\
$\mathrm{H} 3 \mathrm{O}$ & $0.458(14)$ & $0.672(14)$ & $0.689(3)$ & $0.08(3)^{*}$ \\
$\mathrm{H} 4 \mathrm{O}$ & $1.016(7)$ & $0.543(16)$ & $0.666(3)$ & $0.05(2)^{*}$ \\
$\mathrm{H} 5 \mathrm{O}$ & $0.246(8)$ & $-0.013(12)$ & $0.601(2)$ & $0.037(17)^{*}$ \\
$\mathrm{H} 6 \mathrm{O}$ & $0.829(11)$ & $-0.166(10)$ & $0.585(2)$ & $0.033(16)^{*}$ \\
& & & &
\end{tabular}

Atomic displacement parameters $\left(\AA^{2}\right)$

\begin{tabular}{lllllll}
\hline & $U^{11}$ & $U^{22}$ & $U^{33}$ & $U^{12}$ & $U^{13}$ & $U^{23}$ \\
\hline C1 & $0.0066(14)$ & $0.0104(15)$ & $0.0125(15)$ & $-0.0002(11)$ & $-0.0005(11)$ & $0.0021(11)$ \\
O1 & $0.0092(11)$ & $0.0132(12)$ & $0.0146(12)$ & $-0.0038(9)$ & $0.0000(9)$ & $0.0016(9)$ \\
C2 & $0.0068(13)$ & $0.0078(14)$ & $0.0130(14)$ & $-0.0009(11)$ & $-0.0001(11)$ & $0.0005(11)$ \\
O2 & $0.0077(11)$ & $0.0113(11)$ & $0.0145(11)$ & $0.0026(9)$ & $-0.0005(9)$ & $0.0014(9)$ \\
C3 & $0.0094(14)$ & $0.0106(15)$ & $0.0108(14)$ & $-0.0007(11)$ & $-0.0013(11)$ & $-0.0001(11)$
\end{tabular}




\begin{tabular}{lllllll} 
O3 & $0.0094(10)$ & $0.0124(12)$ & $0.0152(11)$ & $0.0009(10)$ & $-0.0010(8)$ & $0.0033(10)$ \\
C4 & $0.0090(14)$ & $0.0105(15)$ & $0.0122(14)$ & $0.0013(12)$ & $0.0005(11)$ & $0.0017(11)$ \\
O4 & $0.0086(11)$ & $0.0134(12)$ & $0.0173(12)$ & $-0.0033(9)$ & $-0.0019(9)$ & $0.0022(9)$ \\
C5 & $0.0120(15)$ & $0.0113(15)$ & $0.0129(15)$ & $-0.0011(12)$ & $-0.0006(12)$ & $0.0012(12)$ \\
O5 & $0.0117(12)$ & $0.0154(13)$ & $0.0171(12)$ & $-0.0043(10)$ & $-0.0023(9)$ & $0.0032(10)$ \\
C6 & $0.0164(16)$ & $0.0139(18)$ & $0.0118(14)$ & $0.0017(12)$ & $-0.0016(12)$ & $0.0001(11)$ \\
O6 & $0.0133(12)$ & $0.0151(14)$ & $0.0167(13)$ & $0.0004(10)$ & $0.0024(9)$ & $0.0002(10)$ \\
S1 & $0.0081(3)$ & $0.0080(3)$ & $0.0112(3)$ & $0.0010(3)$ & $-0.0003(2)$ & $-0.0002(3)$ \\
O11 & $0.0138(12)$ & $0.0150(12)$ & $0.0128(11)$ & $0.0059(10)$ & $0.0007(9)$ & $-0.0018(10)$ \\
O12 & $0.0150(12)$ & $0.0093(11)$ & $0.0176(12)$ & $0.0038(9)$ & $-0.0033(9)$ & $0.0012(9)$ \\
O13 & $0.0104(11)$ & $0.0100(11)$ & $0.0171(12)$ & $-0.0031(9)$ & $-0.0002(9)$ & $0.0000(9)$ \\
$\mathrm{Na}$ & $0.0122(6)$ & $0.0123(7)$ & $0.0138(6)$ & $0.0036(5)$ & $-0.0006(5)$ & $-0.0009(5)$ \\
\hline
\end{tabular}

Geometric parameters $\left(\AA,{ }^{\circ}\right)$

\begin{tabular}{|c|c|c|c|}
\hline $\mathrm{Na}-\mathrm{O} 11^{\mathrm{i}}$ & $2.293(3)$ & $\mathrm{O} 4-\mathrm{H} 4 \mathrm{O}$ & $0.82(3)$ \\
\hline $\mathrm{Na}-\mathrm{O} 12^{\mathrm{ii}}$ & $2.328(3)$ & $\mathrm{C} 5-\mathrm{O} 5$ & $1.424(4)$ \\
\hline $\mathrm{Na}-\mathrm{O} 11^{\mathrm{iii}}$ & $2.370(3)$ & $\mathrm{C} 5-\mathrm{C} 6$ & $1.527(5)$ \\
\hline $\mathrm{Na}-\mathrm{O} 1$ & $2.386(3)$ & $\mathrm{C} 5-\mathrm{H} 5$ & $1.08(5)$ \\
\hline $\mathrm{Na}-\mathrm{O} 13^{\text {iv }}$ & $2.421(3)$ & $\mathrm{O} 5-\mathrm{H} 5 \mathrm{O}$ & $0.80(3)$ \\
\hline $\mathrm{Na}-\mathrm{O} 12^{\mathrm{iii}}$ & $2.757(3)$ & $\mathrm{C} 6-\mathrm{O} 6$ & $1.434(4)$ \\
\hline $\mathrm{C} 1-\mathrm{O} 1$ & $1.415(4)$ & C6-H61 & $1.01(4)$ \\
\hline $\mathrm{C} 1-\mathrm{C} 2$ & $1.537(5)$ & C6-H62 & $0.94(4)$ \\
\hline $\mathrm{C} 1-\mathrm{S} 1$ & $1.802(3)$ & $\mathrm{O} 6-\mathrm{H} 6 \mathrm{O}$ & $0.81(3)$ \\
\hline $\mathrm{C} 1-\mathrm{H} 1$ & $0.94(6)$ & $\mathrm{S} 1-\mathrm{O} 12$ & $1.456(3)$ \\
\hline $\mathrm{O} 1-\mathrm{Na}$ & $2.386(3)$ & $\mathrm{S} 1-\mathrm{O} 11$ & $1.459(3)$ \\
\hline $\mathrm{O} 1-\mathrm{H} 1 \mathrm{O}$ & $0.81(3)$ & $\mathrm{S} 1-\mathrm{O} 13$ & $1.464(3)$ \\
\hline $\mathrm{C} 2-\mathrm{O} 2$ & $1.429(4)$ & $\mathrm{S} 1-\mathrm{Na}^{\mathrm{v}}$ & $3.0557(16)$ \\
\hline $\mathrm{C} 2-\mathrm{C} 3$ & $1.532(4)$ & $\mathrm{O} 11-\mathrm{Na}^{\mathrm{vi}}$ & $2.293(3)$ \\
\hline $\mathrm{C} 2-\mathrm{H} 2$ & $1.02(8)$ & $\mathrm{O} 11-\mathrm{Na}^{\mathrm{v}}$ & $2.370(3)$ \\
\hline $\mathrm{O} 2-\mathrm{H} 2 \mathrm{O}$ & $0.81(3)$ & $\mathrm{O} 12-\mathrm{Na}^{\mathrm{vii}}$ & $2.328(3)$ \\
\hline $\mathrm{C} 3-\mathrm{O} 3$ & $1.435(4)$ & $\mathrm{O} 12-\mathrm{Na}^{\mathrm{v}}$ & $2.757(3)$ \\
\hline $\mathrm{C} 3-\mathrm{C} 4$ & $1.532(5)$ & $\mathrm{O} 13-\mathrm{Na}^{\text {viii }}$ & $2.421(3)$ \\
\hline $\mathrm{C} 3-\mathrm{H} 3$ & $1.08(4)$ & $\mathrm{Na}-\mathrm{S} 1^{\mathrm{iii}}$ & 3.0557 (16) \\
\hline $\mathrm{O} 3-\mathrm{H} 3 \mathrm{O}$ & $0.81(3)$ & $\mathrm{Na}-\mathrm{Na}^{\mathrm{v}}$ & $3.915(2)$ \\
\hline $\mathrm{C} 4-\mathrm{O} 4$ & $1.432(4)$ & $\mathrm{Na}-\mathrm{Na}^{\mathrm{iii}}$ & $3.915(2)$ \\
\hline $\mathrm{C} 4-\mathrm{C} 5$ & $1.529(5)$ & $\mathrm{Na}-\mathrm{H} 1 \mathrm{O}$ & $2.66(6)$ \\
\hline $\mathrm{C} 4-\mathrm{H} 4$ & $0.90(4)$ & & \\
\hline $\mathrm{O} 1-\mathrm{C} 1-\mathrm{C} 2$ & $109.9(3)$ & $\mathrm{C} 1-\mathrm{S} 1-\mathrm{Na}^{\mathrm{v}}$ & $135.30(11)$ \\
\hline $\mathrm{O} 1-\mathrm{C} 1-\mathrm{S} 1$ & $108.2(2)$ & $\mathrm{S} 1-\mathrm{O} 11-\mathrm{Na}^{\mathrm{vi}}$ & $140.39(16)$ \\
\hline $\mathrm{C} 2-\mathrm{C} 1-\mathrm{S} 1$ & $112.8(2)$ & $\mathrm{S} 1-\mathrm{O} 11-\mathrm{Na}^{\mathrm{v}}$ & $103.31(14)$ \\
\hline $\mathrm{O} 1-\mathrm{C} 1-\mathrm{H} 1$ & $109(3)$ & $\mathrm{Na}^{\mathrm{vi}}-\mathrm{O} 11-\mathrm{Na}^{\mathrm{v}}$ & $114.20(11)$ \\
\hline $\mathrm{C} 2-\mathrm{C} 1-\mathrm{H} 1$ & $109(3)$ & $\mathrm{S} 1-\mathrm{O} 12-\mathrm{Na}^{\mathrm{vii}}$ & $153.71(18)$ \\
\hline $\mathrm{S} 1-\mathrm{C} 1-\mathrm{H} 1$ & $109(3)$ & $\mathrm{S} 1-\mathrm{O} 12-\mathrm{Na}^{\mathrm{v}}$ & $87.25(12)$ \\
\hline $\mathrm{C} 1-\mathrm{O} 1-\mathrm{Na}$ & $137.2(2)$ & $\mathrm{Na}^{\mathrm{vii}}-\mathrm{O} 12-\mathrm{Na}^{\mathrm{v}}$ & $113.65(10)$ \\
\hline $\mathrm{C} 1-\mathrm{O} 1-\mathrm{H} 1 \mathrm{O}$ & $99(5)$ & $\mathrm{S} 1-\mathrm{O} 13-\mathrm{Na}^{\mathrm{viii}}$ & $121.11(15)$ \\
\hline $\mathrm{Na}-\mathrm{O} 1-\mathrm{H} 1 \mathrm{O}$ & $101(5)$ & $\mathrm{O} 11^{\mathrm{i}}-\mathrm{Na}-\mathrm{O} 12^{\mathrm{ii}}$ & $170.53(12)$ \\
\hline
\end{tabular}




\begin{tabular}{|c|c|c|c|}
\hline $\mathrm{O} 2-\mathrm{C} 2-\mathrm{C} 3$ & $109.1(3)$ & $\mathrm{O} 11^{\mathrm{i}}-\mathrm{Na}-\mathrm{O} 11^{\mathrm{iii}}$ & $95.09(8)$ \\
\hline $\mathrm{O} 2-\mathrm{C} 2-\mathrm{C} 1$ & $111.4(3)$ & $\mathrm{O} 12^{\mathrm{ii}}-\mathrm{Na}-\mathrm{O} 11^{\mathrm{iii}}$ & $94.13(11)$ \\
\hline $\mathrm{C} 3-\mathrm{C} 2-\mathrm{C} 1$ & $110.5(3)$ & 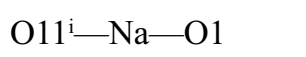 & $91.47(10)$ \\
\hline $\mathrm{O} 2-\mathrm{C} 2-\mathrm{H} 2$ & $114(3)$ & $\mathrm{O} 12^{\mathrm{ii}}-\mathrm{Na}-\mathrm{O} 1$ & $85.52(10)$ \\
\hline $\mathrm{C} 3-\mathrm{C} 2-\mathrm{H} 2$ & $111(3)$ & 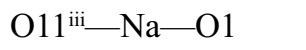 & $121.61(11)$ \\
\hline $\mathrm{C} 1-\mathrm{C} 2-\mathrm{H} 2$ & $101(3)$ & $\mathrm{O} 11^{\mathrm{i}}-\mathrm{Na}-\mathrm{O} 13^{\mathrm{iv}}$ & $88.09(10)$ \\
\hline $\mathrm{C} 2-\mathrm{O} 2-\mathrm{H} 2 \mathrm{O}$ & $106(4)$ & $\mathrm{O} 12^{\mathrm{ii}}-\mathrm{Na}-\mathrm{O} 13^{\mathrm{iv}}$ & $82.76(10)$ \\
\hline $\mathrm{O} 3-\mathrm{C} 3-\mathrm{C} 4$ & $111.1(3)$ & 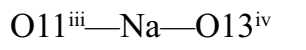 & $151.65(11)$ \\
\hline $\mathrm{O} 3-\mathrm{C} 3-\mathrm{C} 2$ & $109.1(3)$ & $\mathrm{O} 1-\mathrm{Na}-\mathrm{O} 13^{\mathrm{iv}}$ & $86.37(10)$ \\
\hline $\mathrm{C} 4-\mathrm{C} 3-\mathrm{C} 2$ & $111.5(3)$ & 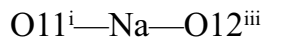 & $90.22(10)$ \\
\hline $\mathrm{O} 3-\mathrm{C} 3-\mathrm{H} 3$ & $101(2)$ & 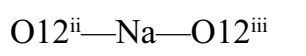 & $93.28(8)$ \\
\hline $\mathrm{C} 4-\mathrm{C} 3-\mathrm{H} 3$ & $113(2)$ & $\mathrm{O} 11^{\mathrm{iii}}-\mathrm{Na}-\mathrm{O} 12^{\mathrm{iii}}$ & $55.17(9)$ \\
\hline $\mathrm{C} 2-\mathrm{C} 3-\mathrm{H} 3$ & $111(2)$ & $\mathrm{O} 1-\mathrm{Na}-\mathrm{O} 12^{\mathrm{iii}}$ & $176.51(11)$ \\
\hline $\mathrm{C} 3-\mathrm{O} 3-\mathrm{H} 3 \mathrm{O}$ & $100(6)$ & $\mathrm{O} 13^{\mathrm{iv}}-\mathrm{Na}-\mathrm{O} 12^{\mathrm{iii}}$ & $96.74(10)$ \\
\hline $\mathrm{O} 4-\mathrm{C} 4-\mathrm{C} 5$ & $106.6(3)$ & $\mathrm{O} 11^{\mathrm{i}}-\mathrm{Na}-\mathrm{S} 1^{\mathrm{iii}}$ & $98.35(8)$ \\
\hline $\mathrm{O} 4-\mathrm{C} 4-\mathrm{C} 3$ & $109.5(3)$ & $\mathrm{O} 12^{\mathrm{ii}}-\mathrm{Na}-\mathrm{S} 1^{\mathrm{iii}}$ & $88.84(8)$ \\
\hline $\mathrm{C} 5-\mathrm{C} 4-\mathrm{C} 3$ & $112.1(3)$ & $\mathrm{O} 11^{\mathrm{iii}}-\mathrm{Na}-\mathrm{S} 1^{\mathrm{iii}}$ & $27.68(7)$ \\
\hline $\mathrm{O} 4-\mathrm{C} 4-\mathrm{H} 4$ & $109(3)$ & $\mathrm{O} 1-\mathrm{Na}-\mathrm{S} 1^{\mathrm{iii}}$ & $148.15(9)$ \\
\hline $\mathrm{C} 5-\mathrm{C} 4-\mathrm{H} 4$ & $110(3)$ & $\mathrm{O} 13^{\mathrm{iv}}-\mathrm{Na}-\mathrm{S} 1^{\mathrm{iii}}$ & $123.98(8)$ \\
\hline $\mathrm{C} 3-\mathrm{C} 4-\mathrm{H} 4$ & $109(3)$ & 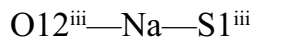 & $28.43(6)$ \\
\hline $\mathrm{C} 4-\mathrm{O} 4-\mathrm{H} 4 \mathrm{O}$ & $112(5)$ & $\mathrm{O} 11^{\mathrm{i}}-\mathrm{Na}-\mathrm{Na}^{\mathrm{v}}$ & $96.85(9)$ \\
\hline $\mathrm{O} 5-\mathrm{C} 5-\mathrm{C} 6$ & $109.3(3)$ & $\mathrm{O} 12^{\mathrm{ii}}-\mathrm{Na}-\mathrm{Na}^{\mathrm{v}}$ & $92.10(7)$ \\
\hline $\mathrm{O} 5-\mathrm{C} 5-\mathrm{C} 4$ & $107.3(3)$ & $\mathrm{O} 11^{\mathrm{iii}}-\mathrm{Na}-\mathrm{Na}^{\mathrm{v}}$ & $32.29(7)$ \\
\hline $\mathrm{C} 6-\mathrm{C} 5-\mathrm{C} 4$ & $112.5(3)$ & $\mathrm{O} 1-\mathrm{Na}-\mathrm{Na}^{\mathrm{v}}$ & $89.32(8)$ \\
\hline $\mathrm{O} 5-\mathrm{C} 5-\mathrm{H} 5$ & $105(3)$ & $\mathrm{O} 13^{\mathrm{iv}}-\mathrm{Na}-\mathrm{Na}^{\mathrm{v}}$ & $173.53(8)$ \\
\hline $\mathrm{C} 6-\mathrm{C} 5-\mathrm{H} 5$ & $112(3)$ & 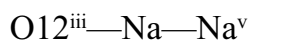 & $87.45(8)$ \\
\hline $\mathrm{C} 4-\mathrm{C} 5-\mathrm{H} 5$ & $111(3)$ & 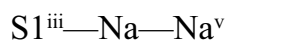 & $59.55(4)$ \\
\hline $\mathrm{C} 5-\mathrm{O} 5-\mathrm{H} 5 \mathrm{O}$ & $105(4)$ & $\mathrm{O} 11^{\mathrm{i}}-\mathrm{Na}-\mathrm{Na}^{\mathrm{iii}}$ & $33.52(6)$ \\
\hline $\mathrm{O} 6-\mathrm{C} 6-\mathrm{C} 5$ & $112.4(3)$ & $\mathrm{O} 12^{\mathrm{ii}}-\mathrm{Na}-\mathrm{Na}^{\mathrm{iii}}$ & $153.15(10)$ \\
\hline $\mathrm{O} 6-\mathrm{C} 6-\mathrm{H} 61$ & $111(2)$ & $\mathrm{O} 11^{\mathrm{iii}}-\mathrm{Na}-\mathrm{Na}^{\mathrm{iii}}$ & $65.29(9)$ \\
\hline $\mathrm{C} 5-\mathrm{C} 6-\mathrm{H} 61$ & $107(2)$ & $\mathrm{O} 1-\mathrm{Na}-\mathrm{Na}^{\mathrm{iii}}$ & $119.46(9)$ \\
\hline $\mathrm{O} 6-\mathrm{C} 6-\mathrm{H} 62$ & $109(3)$ & $\mathrm{O} 13^{\mathrm{iv}}-\mathrm{Na}-\mathrm{Na}^{\mathrm{iii}}$ & $106.89(7)$ \\
\hline $\mathrm{C} 5-\mathrm{C} 6-\mathrm{H} 62$ & $114(3)$ & $\mathrm{O} 12^{\mathrm{iii}}-\mathrm{Na}-\mathrm{Na}^{\mathrm{iii}}$ & $61.18(7)$ \\
\hline $\mathrm{H} 61-\mathrm{C} 6-\mathrm{H} 62$ & $104(4)$ & $\mathrm{S} 1^{\mathrm{iii}}-\mathrm{Na}-\mathrm{Na}^{\mathrm{iii}}$ & $64.84(4)$ \\
\hline $\mathrm{C} 6-\mathrm{O} 6-\mathrm{H} 6 \mathrm{O}$ & $111(4)$ & $\mathrm{Na}^{\mathrm{v}}-\mathrm{Na}-\mathrm{Na}^{\mathrm{iii}}$ & $79.45(5)$ \\
\hline $\mathrm{O} 12-\mathrm{S} 1-\mathrm{O} 11$ & $110.74(16)$ & 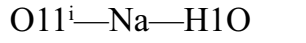 & $102.9(13)$ \\
\hline $\mathrm{O} 12-\mathrm{S} 1-\mathrm{O} 13$ & $113.71(16)$ & $\mathrm{O} 12^{\mathrm{ii}}-\mathrm{Na}-\mathrm{H} 1 \mathrm{O}$ & $72.2(12)$ \\
\hline $\mathrm{O} 11-\mathrm{S} 1-\mathrm{O} 13$ & $113.22(16)$ & 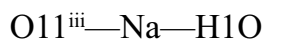 & $132.0(16)$ \\
\hline $\mathrm{O} 12-\mathrm{S} 1-\mathrm{C} 1$ & $108.65(16)$ & $\mathrm{O} 1-\mathrm{Na}-\mathrm{H} 1 \mathrm{O}$ & $17.4(8)$ \\
\hline $\mathrm{O} 11-\mathrm{S} 1-\mathrm{C} 1$ & $105.88(16)$ & $\mathrm{O}_{13} 3^{\mathrm{iv}}-\mathrm{Na}-\mathrm{H} 1 \mathrm{O}$ & $74.0(14)$ \\
\hline $\mathrm{O} 13-\mathrm{S} 1-\mathrm{C} 1$ & $104.03(15)$ & 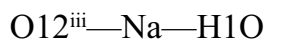 & $163.4(9)$ \\
\hline $\mathrm{O} 12-\mathrm{S} 1-\mathrm{Na}^{\mathrm{v}}$ & $64.32(11)$ & $\mathrm{S} 1^{\mathrm{iii}}-\mathrm{Na}-\mathrm{H} 1 \mathrm{O}$ & $152.7(17)$ \\
\hline $\mathrm{O} 11-\mathrm{S} 1-\mathrm{Na}^{\mathrm{v}}$ & $49.00(11)$ & $\mathrm{Na}^{\mathrm{v}}-\mathrm{Na}-\mathrm{H} 1 \mathrm{O}$ & $100.8(14)$ \\
\hline $\mathrm{O} 13-\mathrm{S} 1-\mathrm{Na}^{\mathrm{v}}$ & $119.44(11)$ & $\mathrm{Na}^{\mathrm{iii}}-\mathrm{Na}-\mathrm{H} 1 \mathrm{O}$ & $134.2(11)$ \\
\hline $\mathrm{C} 2-\mathrm{C} 1-\mathrm{O} 1-\mathrm{Na}$ & $150.0(2)$ & $\mathrm{C} 2-\mathrm{C} 1-\mathrm{S} 1-\mathrm{O} 11$ & $163.8(2)$ \\
\hline $\mathrm{S} 1-\mathrm{C} 1-\mathrm{O} 1-\mathrm{Na}$ & $26.4(4)$ & $\mathrm{O} 1-\mathrm{C} 1-\mathrm{S} 1-\mathrm{O} 13$ & $166.0(2)$ \\
\hline $\mathrm{O} 1-\mathrm{C} 1-\mathrm{C} 2-\mathrm{O} 2$ & $-55.8(3)$ & $\mathrm{C} 2-\mathrm{C} 1-\mathrm{S} 1-\mathrm{O} 13$ & $44.2(3)$ \\
\hline
\end{tabular}




$\begin{array}{llll}\mathrm{S} 1-\mathrm{C} 1-\mathrm{C} 2-\mathrm{O} 2 & 65.0(3) & \mathrm{O} 1-\mathrm{C} 1-\mathrm{S} 1-\mathrm{Na}^{v} & -27.4(3) \\ \mathrm{O} 1-\mathrm{C} 1-\mathrm{C} 2-\mathrm{C} 3 & 65.6(3) & \mathrm{C} 2-\mathrm{C} 1-\mathrm{S} 1-\mathrm{Na}^{v} & -149.19(18) \\ \mathrm{S} 1-\mathrm{C} 1-\mathrm{C} 2-\mathrm{C} 3 & -173.6(2) & \mathrm{O} 12-\mathrm{S} 1-\mathrm{O} 11-\mathrm{Na}^{\mathrm{vi}} & -179.4(2) \\ \mathrm{O} 2-\mathrm{C} 2-\mathrm{C} 3-\mathrm{O} 3 & 179.6(3) & \mathrm{O} 13-\mathrm{S} 1-\mathrm{O} 11-\mathrm{Na}^{\mathrm{vi}} & 51.6(3) \\ \mathrm{C} 1-\mathrm{C} 2-\mathrm{C} 3-\mathrm{O} 3 & 56.8(3) & \mathrm{C} 1-\mathrm{S} 1-\mathrm{O} 11-\mathrm{Na}^{\mathrm{vi}} & -61.8(3) \\ \mathrm{O} 2-\mathrm{C} 2-\mathrm{C} 3-\mathrm{C} 4 & -57.3(3) & \mathrm{Na}-\mathrm{S} 1-\mathrm{O} 11-\mathrm{Na}^{\mathrm{vi}} & 161.2(3) \\ \mathrm{C} 1-\mathrm{C} 2-\mathrm{C} 3-\mathrm{C} 4 & 179.9(3) & \mathrm{O} 12-\mathrm{S} 1-\mathrm{O} 11-\mathrm{Na}^{v} & 19.44(19) \\ \mathrm{O} 3-\mathrm{C} 3-\mathrm{C} 4-\mathrm{O} 4 & 57.3(3) & \mathrm{O} 13-\mathrm{S} 1-\mathrm{O} 11-\mathrm{Na}^{v} & -109.62(15) \\ \mathrm{C} 2-\mathrm{C} 3-\mathrm{C} 4-\mathrm{O} 4 & -64.6(3) & \mathrm{C} 1-\mathrm{S} 1-\mathrm{O} 11-\mathrm{Na}^{v} & 137.03(14) \\ \mathrm{O} 3-\mathrm{C} 3-\mathrm{C} 4-\mathrm{C} 5 & -60.8(4) & \mathrm{O} 11-\mathrm{S} 1-\mathrm{O} 12-\mathrm{Na}^{\mathrm{vii}} & -160.2(4) \\ \mathrm{C} 2-\mathrm{C} 3-\mathrm{C} 4-\mathrm{C} 5 & 177.2(3) & \mathrm{O} 13-\mathrm{S} 1-\mathrm{O} 12-\mathrm{Na}^{\mathrm{vii}} & -31.4(4) \\ \mathrm{O} 4-\mathrm{C} 4-\mathrm{C} 5-\mathrm{O} 5 & -179.7(3) & \mathrm{C} 1-\mathrm{S} 1-\mathrm{O} 12-\mathrm{Na}^{\mathrm{vii}} & 83.9(4) \\ \mathrm{C} 3-\mathrm{C} 4-\mathrm{C} 5-\mathrm{O} 5 & -59.9(4) & \mathrm{Na}-\mathrm{S} 1-\mathrm{O} 12-\mathrm{Na}^{\mathrm{vii}} & -144.0(4) \\ \mathrm{O} 4-\mathrm{C} 4-\mathrm{C} 5-\mathrm{C} 6 & 60.0(4) & \mathrm{O} 11-\mathrm{S} 1-\mathrm{O} 12-\mathrm{Na}^{\mathrm{v}} & -16.19(16) \\ \mathrm{C} 3-\mathrm{C} 4-\mathrm{C} 5-\mathrm{C} 6 & 179.9(3) & \mathrm{O} 13-\mathrm{S} 1-\mathrm{O} 12-\mathrm{Na}^{\mathrm{v}} & 112.62(14) \\ \mathrm{O} 5-\mathrm{C} 5-\mathrm{C} 6-\mathrm{O} 6 & -57.8(4) & \mathrm{C} 1-\mathrm{S} 1-\mathrm{O} 12-\mathrm{Na}^{\mathrm{v}} & -132.07(12) \\ \mathrm{C} 4-\mathrm{C} 5-\mathrm{C} 6-\mathrm{O} 6 & 61.2(4) & \mathrm{O} 12-\mathrm{S} 1-\mathrm{O} 13-\mathrm{Na}^{\mathrm{viii}} & -67.7(2) \\ \mathrm{O} 1-\mathrm{C} 1-\mathrm{S} 1-\mathrm{O} 12 & 44.6(3) & \mathrm{O} 11-\mathrm{S} 1-\mathrm{O} 13-\mathrm{Na}^{\mathrm{viii}} & 59.9(2) \\ \mathrm{C} 2-\mathrm{C} 1-\mathrm{S} 1-\mathrm{O} 12 & -77.2(3) & \mathrm{C} 1-\mathrm{S} 1-\mathrm{O} 13-\mathrm{Na}^{\mathrm{viii}} & 174.31(16) \\ \mathrm{O} 1-\mathrm{C} 1-\mathrm{S} 1-\mathrm{O} 11 & -74.4(3) & \mathrm{Na}-\mathrm{S} 1-\mathrm{O} 13-\mathrm{Na}^{\mathrm{viii}} & 5.1(2) \\ \end{array}$

Symmetry codes: (i) $x, y-1, z$; (ii) $x-1, y, z$; (iii) $-x+1, y-1 / 2,-z+2$; (iv) $x-1, y-1, z$; (v) $-x+1, y+1 / 2,-z+2$; (vi) $x, y+1, z$; (vii) $x+1, y, z$; (viii) $x+1, y+1, z$.

Hydrogen-bond geometry $\left(A,{ }^{\circ}\right)$

\begin{tabular}{lllll}
\hline$D-\mathrm{H} \cdots A$ & $D-\mathrm{H}$ & $\mathrm{H} \cdots A$ & $D \cdots A$ & $D-\mathrm{H}^{\cdots} A$ \\
\hline $\mathrm{O} 1-\mathrm{H} 1 O \cdots \mathrm{O} 2^{\mathrm{ii}}$ & $0.81(3)$ & $1.92(4)$ & $2.693(4)$ & $160(8)$ \\
$\mathrm{O} 2-\mathrm{H} 2 O \cdots \mathrm{O} 1$ & $0.81(3)$ & $2.36(5)$ & $2.828(3)$ & $118(5)$ \\
$\mathrm{O} 2-\mathrm{H} 2 O \cdots \mathrm{O} 13^{\mathrm{i}}$ & $0.81(3)$ & $2.08(4)$ & $2.745(4)$ & $140(5)$ \\
$\mathrm{O} 3-\mathrm{H} 3 O \cdots \mathrm{O} 4$ & $0.81(3)$ & $2.32(7)$ & $2.838(3)$ & $123(7)$ \\
$\mathrm{O} 3-\mathrm{H} 3 O \cdots 5^{\mathrm{vi}}$ & $0.81(3)$ & $2.11(6)$ & $2.743(4)$ & $136(7)$ \\
$\mathrm{O} 4-\mathrm{H} 4 O \cdots 3^{\mathrm{vii}}$ & $0.82(3)$ & $1.87(3)$ & $2.692(3)$ & $179(8)$ \\
$\mathrm{O} 5-\mathrm{H} 5 O \cdots \mathrm{O}^{\mathrm{ii}}$ & $0.80(3)$ & $1.91(3)$ & $2.704(4)$ & $169(6)$ \\
$\mathrm{O} 6-\mathrm{H} 6 O \cdots 4^{\mathrm{i}}$ & $0.81(3)$ & $2.00(4)$ & $2.753(4)$ & $154(6)$ \\
$\mathrm{O} 6-\mathrm{H} 6 O \cdots \mathrm{O} 5$ & $0.81(3)$ & $2.45(6)$ & $2.853(4)$ & $112(5)$ \\
\hline
\end{tabular}

Symmetry codes: (i) $x, y-1, z$; (ii) $x-1, y, z$; (vi) $x, y+1, z$; (vii) $x+1, y, z$. 